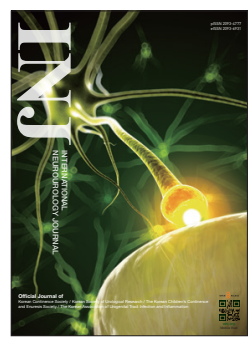

\title{
Pioglitazone Alters the Proteomes of Normal Bladder Epithelial Cells but Shows No Tumorigenic Effects
}

\author{
Muhammad Shahid ${ }^{1, \star}$, Minhyung Kim ${ }^{1, \star}$, Austin Yeon ${ }^{1}$, Peng Jin ${ }^{1}$, Woong-Ki Kim², Sungyong You ${ }^{1,3}$, Jayoung Kim ${ }^{1,3,45}$ \\ ${ }^{1}$ Departments of Surgery and Biomedical Sciences, Cedars-Sinai Medical Center, Los Angeles, CA, USA \\ ${ }^{2}$ Department of Microbiology and Molecular Cell Biology, Eastern Virginia Medical School, Norfolk, VA, USA \\ ${ }^{3}$ Samuel Oschin Comprehensive Cancer Institute, Cedars-Sinai Medical Center, Los Angeles, CA, USA \\ ${ }^{4}$ University of California Los Angeles, Los Angeles, CA, USA \\ ${ }^{5}$ Department of Urology, Ga Cheon University College of Medicine, Incheon, Korea
}

Purpose: Pioglitazone, an antihyperglycemic drug, is widely used in diabetes mellitus patients with insulin resistance. Although pioglitazone is known to have a potential link to bladder cancer (BC), there have been contradictory results. This present study is designed to understand the regulatory mechanisms that drive the effects of pioglitazone on the bladder epithelial cells.

Methods: Labeled liquid chromatography-tandem mass spectrometry-based proteomics profiling characterized the global proteomes of normal human bladder epithelial cells treated with or without pioglitazone.

Results: This approach detected approximately 5,769 proteins in total. Of those 5,769 proteins, 124 were identified as being differentially expressed due to pioglitazone treatment. Further analysis identified 95 upregulated and 29 downregulated proteins (absolute $\log _{2}$ fold change $>0.58$ and P-value $<0.05$ ). The following functional gene enrichment analysis suggested that pioglitazone may be altering a few select biological processes, such as gene/chromatin silencing, by downregulating BMI1 (B lymphoma Mo-MLV insertion region 1 homolog), a polycomb complex protein. Further cell-based assays showed that cell adhesion molecules, epithelial-mesenchymal transition markers, and major signaling pathways were significantly downregulated by pioglitazone treatment.

Conclusions: These experimental results revealed the proteomic and biological alterations that occur in normal bladder cells in response to pioglitazone. These findings provided a landscape how bladder proteome is influenced by pioglitazone, which suggests the potential adverse effects of diabetes drugs and their links to bladder dysfunctions.

Keywords: Urinary bladder neoplasms; BMI1; Diabetes mellitus; Global proteome; Peroxisome proliferator-activated receptor gamma

- Fund/Grant Support: The authors acknowledge support from National Institutes of Health grants (1U01DK103260, 1R01DK100974, U24 DK097154, NIH NCATS UCLA CTSI UL1TR000124), Department of Defense grants (W81XWH-15-1-0415 and W81XWH-19-1-0109), Centers for Disease Controls and Prevention (1U01DP006079), IMAGINE NO IC Research Grant, the Steven Spielberg Discovery Fund in Prostate Cancer Research Career Development Award, and the U.S.-Egypt Science and Technology Joint Fund (to J.K.). J.K. is a former recipient of the Interstitial Cystitis Association Pilot Grant, a Fishbein Family IC Research Grant, New York Academy of Medicine, and Boston Children's Hospital Faculty Development. The funders had no role in the experimental design, data collection, analysis, preparation of the manuscript, or decision to publish. In addition, this article is derived from the Subject Data funded in whole or part by National Academies of Sciences, Engineering, and Medicine (NAS) and The United States Agency for International Development (USAID). Any opinions, findings, conclusions, or recommendations expressed in this article are those of the authors alone, and do not necessarily reflect the views of USAID or NAS.

- Conflict of Interest: No potential conflict of interest relevant to this article was reported.

Corresponding author: Jayoung Kim (D) https://orcid.org/0000-0002-3683-4627 Departments of Surgery and Biomedical Sciences, Cedars-Sinai Medical Center, Davis 50718700 Beverly Blvd., Los Angeles, CA 90048, USA

E-mail: Jayoung.Kim@cshs.org

*Muhammad Shahid and Minhyung Kim contributed equally to this study as cofirst authors.

Submitted: October 15, 2019 / Accepted after revision: November 30, 2019 


\section{INTRODUCTION}

Pioglitazone is one of thiazolidinediones (TZDs), which is currently the only oral drugs with a confirmed antihyperglycemic impact in type 2 diabetes mellitus (T2DM) patients. TZDs is well-known agonists of peroxisome proliferator-activated receptor (PPAR) $\alpha$ and $\gamma$, TZDs are known to positively influence insulin sensitivity and $\beta$-cell function and potentially alter lipid profiles. Pioglitazone is a synthetic ligand of PPAR $\gamma$ and is used as a therapeutic treatment for patients with T2DM. PPAR $\gamma$, a ligand-activated transcription factor, is expressed in white and brown adipose tissues as well as in the urinary bladder [1]. Pioglitazone has also been shown to reduce macrophage infiltration through the activation of PPAR $\gamma$ and induction of apoptotic cell death [2], which can lead to decreased numbers of macrophages in adipose tissues [3]. To examine the impact of PPAR $\gamma$ on bladder cell function, several studies compared the PPAR $\gamma$ levels in normal bladder and bladder cancer (BC) mucosal samples. They found that cell migration and invasion rates are correlated with PPAR $\gamma$ expression in various BC cell lines. Recently, in vitro effects of pioglitazone on normal urothelial epithelium (NUTE) cells and BC (J82) cells were compared [4]. In this paper, pioglitazone inhibited the proliferation and induced apoptosis of NUTE cells, but not J82 cells, suggesting the different effects of pioglitazone on normal bladder cells vs BC cells. In addition, prior studies showed that a primary proangiogenic factor, vascular endothelial growth factor A, is positively regulated by $\operatorname{PPAR} \gamma$ [5]. PPAR $\gamma$ is involved in proliferation and survival through Wnt/ $\beta$-catenin, IGF-I/PI3K/AKT/ mTOR, and mitogen activated protein kinase (MAPK) pathways [6]. Furthermore, PPAR $\gamma$ can regulate the inflammatory response of macrophages through Toll-like receptor 4/nuclear

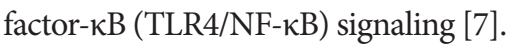

There have been contradictory reports on diabetes mellitus (DM) and elevated risk of BC [8]. Studies have demonstrated that pioglitazone can have adverse effects that exaggerate the risk of BC. DM patients, particularly males, on long-term, high doses of pioglitazone, are at a higher risk of developing BC [9]. The KPNC (Kaiser Permanente Northern California) study determined that inflated dose and length of pioglitazone treatment correlated to increased rates of BC [5]. Currently, the U.S. Food and Drug Administration allows the use of pioglitazone in patients with special caution to those with a prior history or active $\mathrm{BC}[10]$. A potential reason for this observation could be that $\mathrm{PPAR} \gamma$ signaling in $\mathrm{BC}$ cells may provide a tumor micro- environment that allows for de novo lipogenesis of lipids that can be utilized in increasing tumor mass and energy usage [11]. However, other studies have shown that TZDs posed no risk on survival in BC patients who underwent radical cystectomy [12]. A recent meta-analysis found no difference in $\mathrm{BC}$ incidence among users of pioglitazone and nonusers [13]. These inconsistencies between studies have led to questions in the legitimacy of studies and the actual dangers of continued pioglitazone use. Due to the inherent difficulty of accurately assessing the link between TZDs and $\mathrm{BC}$, the association between the two is still in controversy [14].

The goal of our study was to determine whether TZDs have any negative or positive effects on the healthy bladder epithelium. Since pathophysiological changes require the actions of a series of key proteins, global proteomic analysis of protein alterations using state-of-the-art mass spectrometry is ideal for acquiring unbiased biological information regarding molecular mechanisms. Using such a method, this study aimed to understand the mechanistic meanings of proteome perturbations in the normal bladder due to TZD treatment. We sought to examine the effects of pioglitazone specifically, being that is the most widely used TZD. Our global quantitative proteomic analyses found that pioglitazone alters the proteome and biological networks in normal human bladder cells without tumorigenic effects.

\section{MATERIALS AND METHODS}

\section{Cell Culture}

We used human telomerase reverse transcriptase-immortalized human urothelial cell line (TRT-HU1) for this study [15]. Cell lines that were initially established, frozen, and under a passage number of 10 were used in this study. Culture media was changed after one day of subculturing and cells were passed again when there was $70 \%-80 \%$ confluence. All cells were negative for mycoplasma contamination. For maintaining TRTHU1, we used Dulbecco's modified Eagle's medium supplemented with $10 \%$ fetal bovine serum (Invitrogen, Waltham, MA, USA), 1\% penicillin/streptomycin, and 1\% L-glutamine (Sigma-Aldrich, St. Louis, MO, USA) under a humidified atmosphere of $5 \% \mathrm{CO}_{2}$ at $37^{\circ} \mathrm{C}$.

\section{Antibodies and Reagents}

Commercial antibodies were obtained from the following sources: actin gamma 2 (ACTG2) (ab189385), myosin heavy chain 3 (MYH3) (ab124205), oxidative phosphorylation (OX- 
PHOS) (ab110413) from Abcam; phospho-NF- $\mathrm{B}$ (3033), phospho-HER2/ErbB2 (2247), phospho-p21 activated kinase 1 (PAK1) (2601), phospho-glycogen synthase kinase-3 $\beta$ (GSK-3 $\beta$ ) (9336), Snail (3879), N-cadherin (13116), Slug (9585), $\beta$-catenin (8480), E-cadherin (14472), Tight Junction Antibody Kit (8683) from Cell Signaling Technology, and $\beta$-actin (A1978) from Sigma. Horseradish peroxidase (HRP)-conjugated secondary antibodies $(7074,7076)$ were purchased from Cell Signaling Technologies. Most of other chemical reagents were procured from Sigma Chemical Corp.

\section{Quantitative Proteomics}

For this study, we performed the tandem mass tagging (TMT)based analysis as we described in previous papers [15]. In brief, cellular proteins from $25 \mu \mathrm{M}$ pioglitazone-treated and control TRT-HU1 cells were extracted using a $4 \%$ sodium dodecyl sulfate-containing buffer. The protein concentration was measured using the Pierce 660nm Assay Kit (Thermo Fisher Scientific, Waltham, MA, USA). The kit was carried out using $60 \mu \mathrm{g}$ of protein from each sample. Samples were digested with trypsin via filter-aided sample preparation and labeled with TMT6plex reagents in parallel. Then, the peptides were reconstituted and desalted via $\mathrm{C} 18$ spin columns (Thermo Fisher Scientific). HighpH reversed-phase liquid chromatography using an Ultimate 3000 XRS System (Thermo Fisher Scientific) was used to separate samples. Peptides with $>30 \%$ precursor ion interference were excluded to minimize inaccurate quantifications caused by precursor ion interference and Proteome Discoverer was used.

\section{Identification of Differentially Expressed Proteins}

Proteins that have more than 3 nonredundant peptides in each sample were selected for further analysis using a one sample ttest using the $\log _{2}$ fold changes to compute the significance. False discovery rates (FDRs) of each protein for the 1 sample ttest were then calculated using Storey's correction method. Differentially expressed proteins (DEPs) were identified based on FDR $<0.05$ and absolute $\log _{2}$ fold change $\geq 0.58$ ( 1.5 -fold). DAVID ver. 6.8 was used for functional enrichment analysis of DEPs. Significantly enriched cellular processes were selected $(\mathrm{P}<0.05)$. Functional classification analysis was performed using PANTHER ver. 11.

\section{Master Regulator Analysis}

To identify master regulators, we discerned each DEP and counted their individual partners. The protein-protein interac- tion information was based on the following 6 interactome databases: the Biological General Repository for Interaction Datasets (BioGRID), the Database of Interacting Proteins (DIP), High confidence protein-protein interactions (HitPredict), the IntAct molecular interaction database (IntAct), the Molecular INTeraction database (MINT), and the STRING database. Fisher exact test was used to calculate the enrichment P-values using. Finally, we selected proteins with P-values lower than 0.01 and prioritized the DEPs based on the number of interaction partners.

\section{Western Blot Analysis}

For western blot analysis we used whole-cell lysates. All primary antibodies incubations were done at $4^{\circ} \mathrm{C}$ overnight. HRPconjugated secondary antibody incubations were done at room temperature for 1 hour. All western blot experiments were repeated at least 3 times for each antibody and analyzed from different lysates using standard procedures.

\section{Quantification of Reactive Oxygen Species}

We measured intracellular reactive oxygen species (ROS) levels by staining with $2^{\prime}, 7^{\prime}$-dichlorofluorescin diacetate (DCF-DA) and sorting with a flow cytometer (FACSCalibur, Becton-Dickinson, Franklin Lakes, NJ, USA). DCF fluorescence intensity was measured with maximum excitation and emission spectra of $495 \mathrm{~nm}$ and $529 \mathrm{~nm}$, respectively.

\section{Cell Proliferation Assay}

To determine cell growth during exposure to pioglitazone, TRT-HU1 were seeded in 6-well plates $\left(5 \times 10^{4}\right.$ cells/well). After treatment with varying doses of pioglitazone $(0,25,50$, or $100 \mu \mathrm{M})($ E6910, Sigma-Aldrich) or vehicle, cell numbers were evaluated after 24, 48, and 72 hours. For crystal violet staining, cells were stained with $0.05 \%$ solution of crystal violet. After incubation at room temperature for 15 minutes, the wells were washed thoroughly with phosphate buffered saline and fixed with $4 \%$ paraformaldehyde at room temperature for additional 5 minutes. For quantitative analysis, $10 \%$ acetic acid solution was used to dissolve the stained cells and absorbance at 570$590 \mathrm{~nm}$ were measured. All the data are representative of $3 \mathrm{in}$ dependent trials.

\section{Statistical Analysis}

In order to evaluate differential expression of the proteins between the 2 groups, we used Student t-tests. Variables with nor- 
mal distribution were expressed as mean \pm standard deviation. All reported $\mathrm{P}$-values are 2-tailed, with $\mathrm{P}<0.05$ of significance.

\section{RESULTS}

\section{Pioglitazone Perturbs the Proteome of Normal Bladder Epithelial Cells}

Labeled LC-MS/MS proteomic analysis was conducted to identify the global proteomes of TRT-HU1 bladder cells treated with or without $25 \mu \mathrm{M}$ of pioglitazone. We found that pioglitazone treatment increased PPAR $\gamma$ protein expression (Fig. 1A). Our proteomics analysis workflow is summarized in Fig. $1 \mathrm{~B}$.

We detected 5,769 proteins in total, including proteins from the cell part $(n=2,375)$, organelle $(n=1,663)$, macromolecular complex $(n=1,060)$, membrane $(n=643)$, extracellular region $(n=88)$, cell junction $(n=61)$, synapse $(n=43)$, and extracellular matrix ( $n=29)$ (Fig. 2A). To identify DEPs between the control versus pioglitazone groups, we performed a statistical hypothesis test using an empirical null model (see Methods). Among the 5,769 proteins, 124 DEPs (including 95 upregulated and 29 downregulated proteins) were found to have absolute $\log _{2}$ foldchanges $\geq 0.58$ and P-values $<0.05$. Approximately $70 \%$ of the DEPs $(n=95)$ showed increased expression due to pioglitazone treatment (Fig. 2B).

Volcano plot displays DEPs between control and pioglitazone groups. The top 5 most significantly up- or downregulated proteins were highlighted with their official symbol (Fig. 2C). HIST1H2BK (histone cluster 1 H2B family member K), ST13P5 (ST13, Hsp70 interacting protein pseudogene 5), and WASH2P (WAS protein family homolog 2 pseudogene) were the most significantly downregulated by pioglitazone treatment. ATPAF1 (ATP synthase mitochondrial F1 complex as-

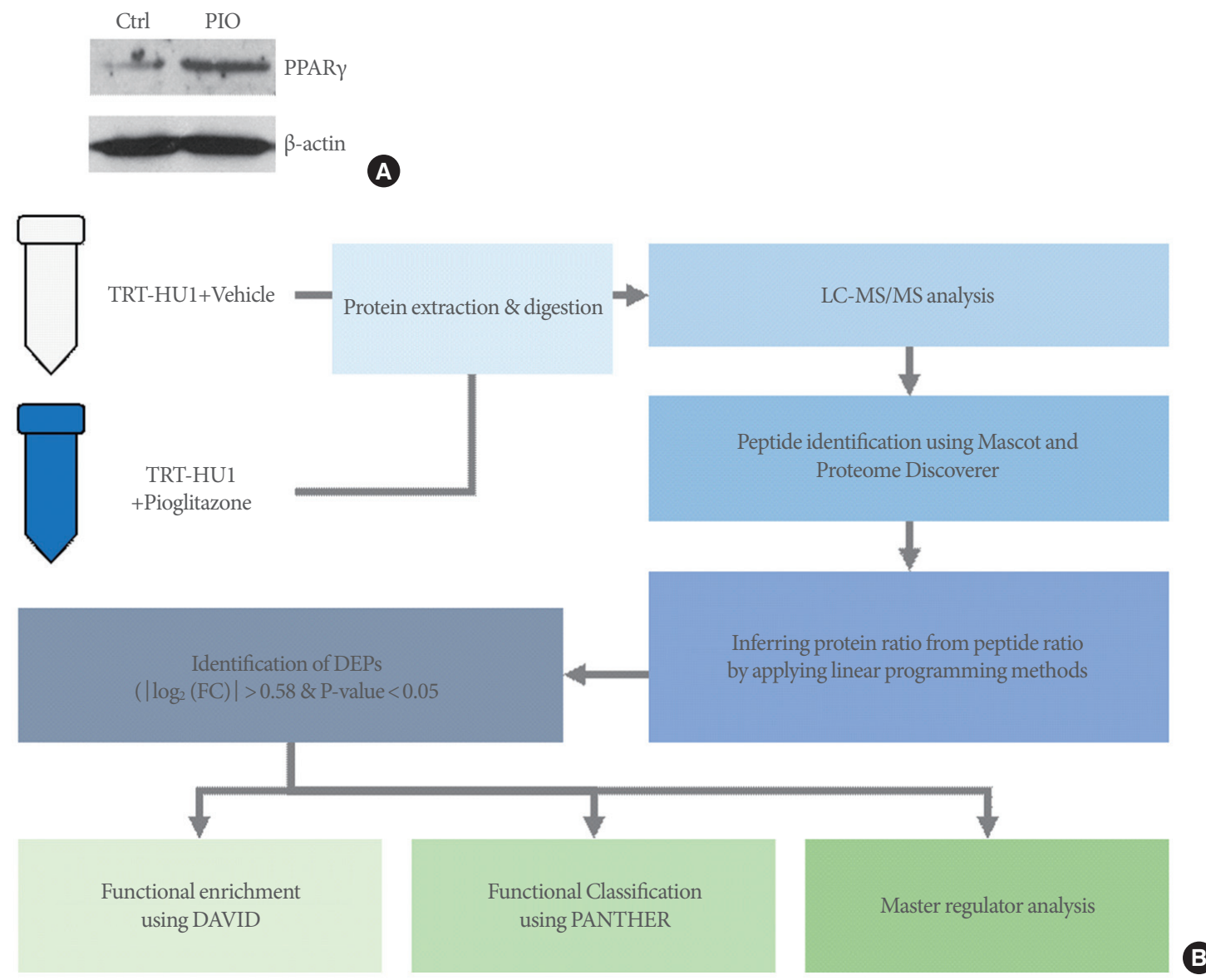

Fig. 1. Overview of global proteomics workflow for data generation and analysis. (A) Western blot analysis shows the effects of pioglitazone treatment on PPAR $\gamma$ expression. (B) Proteomics analysis workflow for this study. Ctrl, control; PIO, pioglitazone treatment; PPAR $\gamma$, peroxisome proliferator-activated receptor gamma; TRT-HU1, human telomerase reverse transcriptase-immortalized human urothelial cells; LC-MS, liquid chromatography-mass spectrometry; DEP, differentially expressed protein; FC, fold change. 

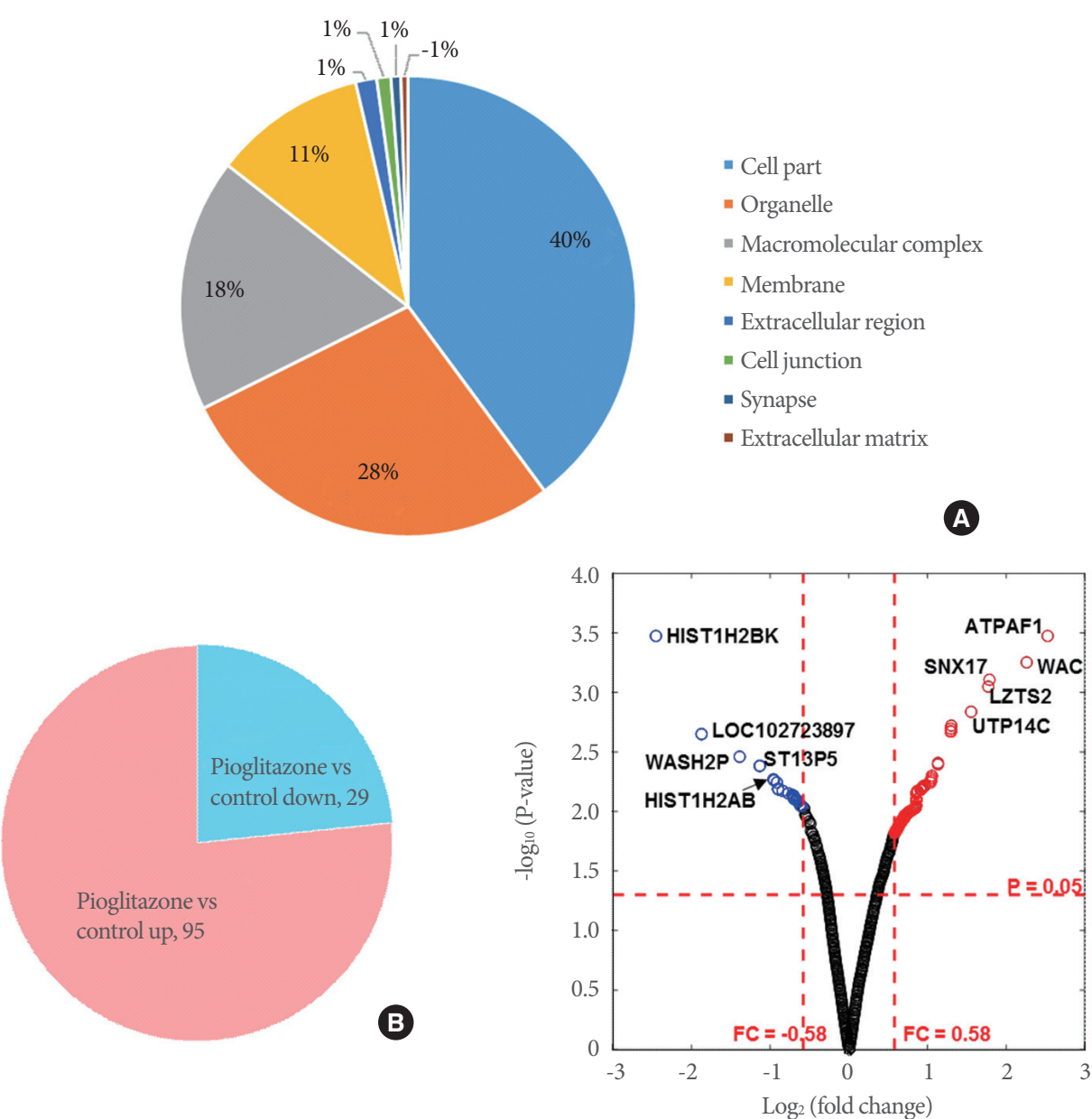

C

Fig. 2. Total proteins and differentially expressed proteins (DEPs) perturbed in response to pioglitazone treatment. (A) Pie chart depicts the cellular location of the detected DEPs. (B) Pie chart shows proportion of up- and downregulated DEPs in cells treated with pioglitazone. (C) A volcano plot showing the up- or downregulated DEPs by pioglitazone treatment. Red, upregulated DEPs; blue, downregulated DEPs. Protein symbol of the top 5 up- or downregulated proteins are presented in the volcano plot. HIST1H2BK, histone cluster $1 \mathrm{H} 2 \mathrm{~B}$ family member K; WASH2P, WAS protein family homolog 2 pseudogene; ST13P5, ST13, Hsp70 interacting protein pseudogene 5; HIST1H2AB, histone H2A type 1-B; ATPAF1, ATP synthase mitochondrial F1 complex assembly factor 1; SNX17, sorting nexin 17; WAC, WW domain containing adaptor with coiled-coil; LZTS2, leucine zipper tumor suppressor 2; UTP14C, small subunit processome component; FC, fold change.

sembly factor 1), SNX17 (sorting nexin 17), LZTS2 (leucine zipper tumor suppressor 2), and UTP14C (UTP14C, small subunit processome component) were the most upregulated in response to pioglitazone. Supplementary Table 1 shows the list of DEPs that were altered in response to pioglitazone along with the transcriptional targets of PPAR $\gamma$ and test statistics. This data suggest that pioglitazone may induce transcriptional programming through PPAR $\gamma$, which contributes to increased protein expression.

\section{Functional Enrichment Analysis Reveals That Pioglitazone Modulates Key Biological Processes}

To check the function of proteins perturbed by pioglitazone, we performed functional enrichment and classification using DAVID and PANTHER, respectively. Muscle filament sliding, actin-mediated cell contraction, cellular oxidant detoxification, cell adhesion, purine nucleoside monophosphate metabolic process, ATP metabolic process, and cytoskeleton organization were enriched by upregulated DEPs. However, chromatin silencing, gene silencing, and regulation of gene expression were enriched by downregulated DEPs (Fig. 3A). We listed the DEPs 
Table 1. Enriched biological processes and corresponding proteins in upregulated and downregulated proteins

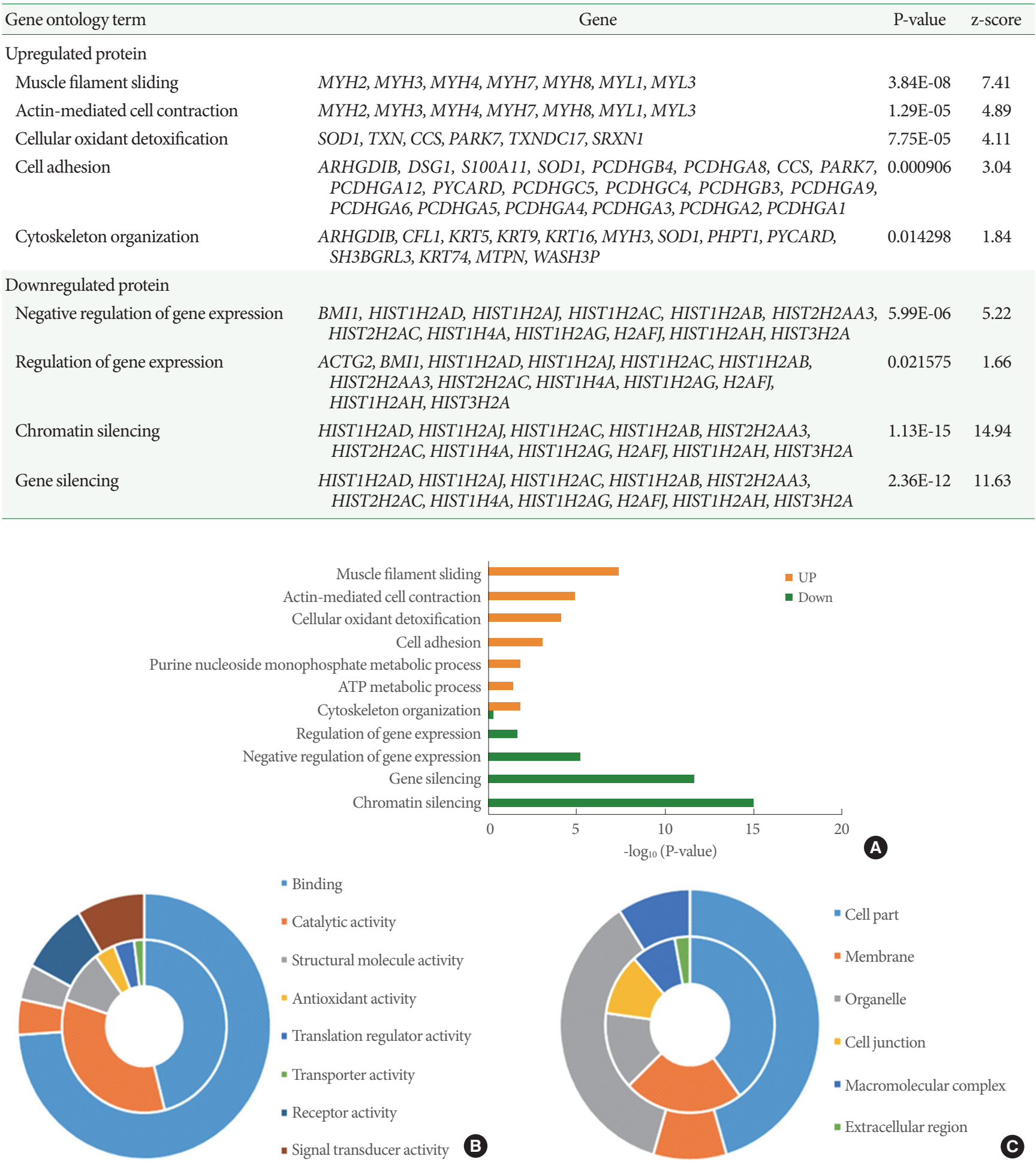

Fig. 3. Functional enrichment and classification of the differentially expressed proteins (DEPs). (A) Bar plot shows biological processes enriched by the up- and downregulated DEPs. (B) Top molecular functions of upregulated (inner circle) and downregulated (outer circle) proteins. (C) Top cellular compartments of upregulated (inner circle) and downregulated (outer circle) proteins. ATP, adenosine triphosphate. 
belonging to actin-mediated cell contraction, cellular oxidant detoxification, cell adhesion, purine nucleoside monophosphate metabolic process, and chromatin silencing (Table 1).

Interestingly, the classified molecular functions of the DEPs include binding, catalytic activity, structural molecule activity, antioxidant activity, translation regulator activity, transporter activity, receptor activity, and signal transducer activity (Fig. 3B). Binding function was the largest portion of both the upregulated and downregulated DEPs. Catalytic, antioxidant, translation regulator, and transporter activity were the major molecular functions in the upregulated DEPs. However, receptor and signal transducer activity were the major functions in downregulated DEPs. The classified cellular compartments of the DEPs were cell part, membrane, organelle, cell junction, macromolecular complex, and extracellular region (Fig. 3C). Membrane, cell junction, and extracellular region were the major cellular compartments in upregulated DEPs. Organelle and macromolecular complex are major cellular compartments in the outer circle.

\section{Identification of Protein Interaction Networks Regulated by Pioglitazone Treatment}

Our present study revealed a large set of proteins $(n=5,769)$ with relevant levels of abundance in normal bladder epithelial cells. Their functions are related to various cellular processes, including actin regulation and chromatin remodeling (Fig. 3A). Pioglitazone perturbs and modulates these cellular processes by affecting protein-protein interactions. We therefore performed master regulator analysis using all the proteins detected in our global proteomic analysis to identify key proteins that can be major nodes in the protein-protein interaction networks. To select key proteins, we first identified proteins with a large number of interactions from those detected. We then determined the top 5 proteins with the largest number of interactions and the most significant P-values (Fig. 4A, Supplementary Table 2).

To reconstruct the network model, top 3 proteins were then used. Of note, B lymphoma Mo-MLV insertion region 1 homo$\log$ (BMI1), Histone H4A (HIST1H4A), and superoxide dismutase 1 (SOD1) were found to be the top 3 regulators, with Pvalues $<0.01$ and large target counts. The network model describes the interactions among the top 3 regulators and their interactors with significant differential expression when treated with pioglitazone. Pioglitazone treatment downregulated expression of BMI1, which was found to interact mainly with histone proteins, such as Histone $\mathrm{H} 2$ (HIST2A), Histone H2A type 3-A (H2AA3), Histone H2A type 2-C (HIST2H2AC), Histone H1
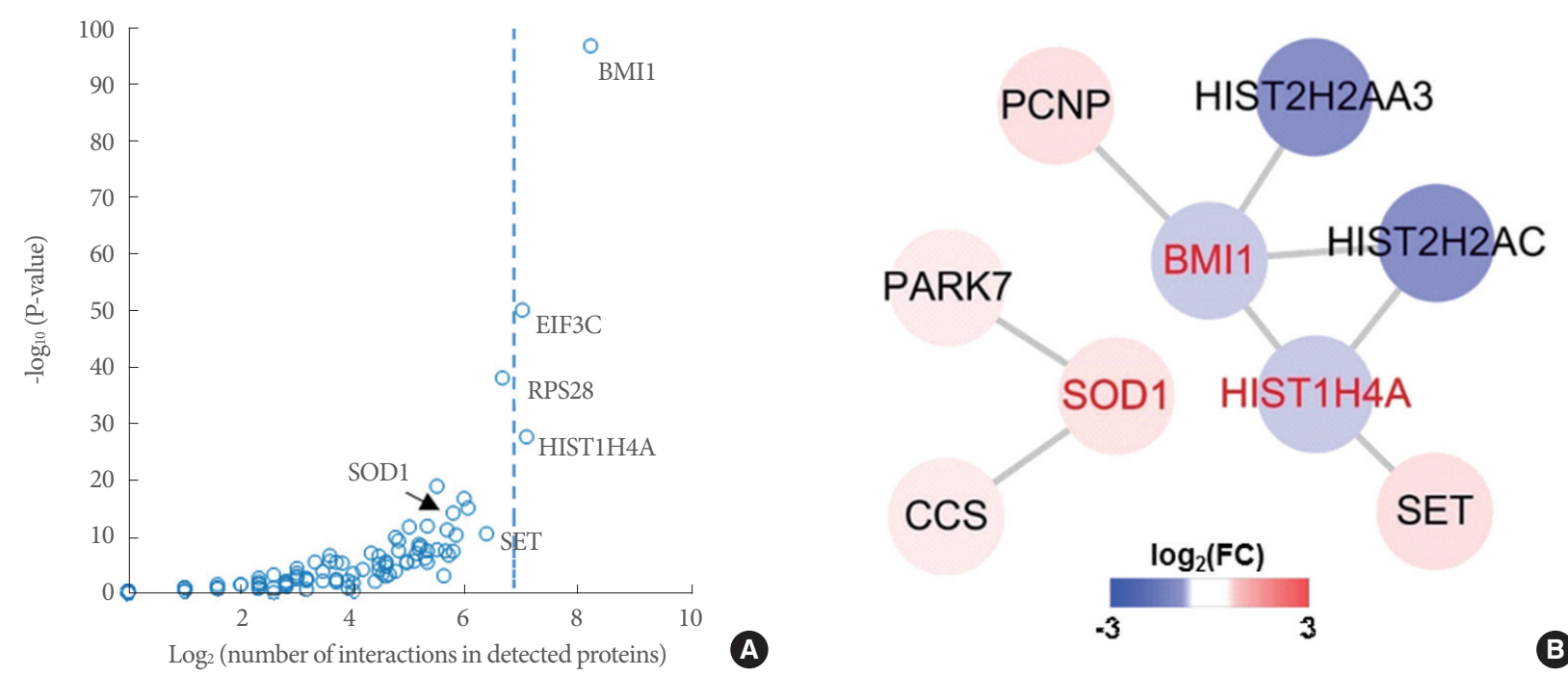

Fig. 4. Potential master regulators suggested by master regulator analysis (MRA). (A) Scatter plot displays distribution of detected proteins by number of interactions and level of significance driven by MRA. (B) Network model describing the interactions of the top 3 master regulators and their interacting differentially expressed protein partners. Red font denotes the top 3 master regulators. BMI1, B lymphoma Mo-MLV insertion region 1 homolog; EIF3C, eukaryotic translation initiation factor 3 subunit C; RPS28, ribosomal protein S28; HIST1H4A, histone cluster $1 \mathrm{H} 4$ family member A; SOD1, superoxide dismutase 1; SET, SET nuclear proto-oncogene; PCNP, PEST proteolytic signal containing nuclear protein; HIST2H2AA3, Histone H2A type 2-A; PARK7, parkinsonism associated deglycase; HIST2H2AC, Histone H2A type 2-C; CCS, copper chaperone for superoxide dismutase; FC, fold change. 
type 4-A (HIST1H4A) (Fig. 4B). Expression of SOD1 increased with pioglitazone treatment and it was found to interact with 2 other upregulated proteins, PARK7 (parkinsonism associated deglycase) and CCS (copper chaperone for superoxide dismutase). Interestingly, downregulation of BMI1 and its interacting histone proteins in the network model suggests potential epigenetic regulation by pioglitazone, which is consistent with functional enrichment data indicating "chromatin silencing" (Fig. 3A).

\section{Biological Influences of Pioglitazone on Normal Bladder Epithelial Cells}

To further validate the DEPs between the control versus pioglitazone groups, proteins were selected for based on functional enrichment analyses. Western blot analysis showed that expression of MYH3 was upregulated, while expression of ACTG2 was downregulated in cells treated with $25 \mu \mathrm{M}$ of pioglitazone (Fig. 5A). Consistent with morphological changes in pioglitazone-treated cells, we noticed that protein expression of mes-
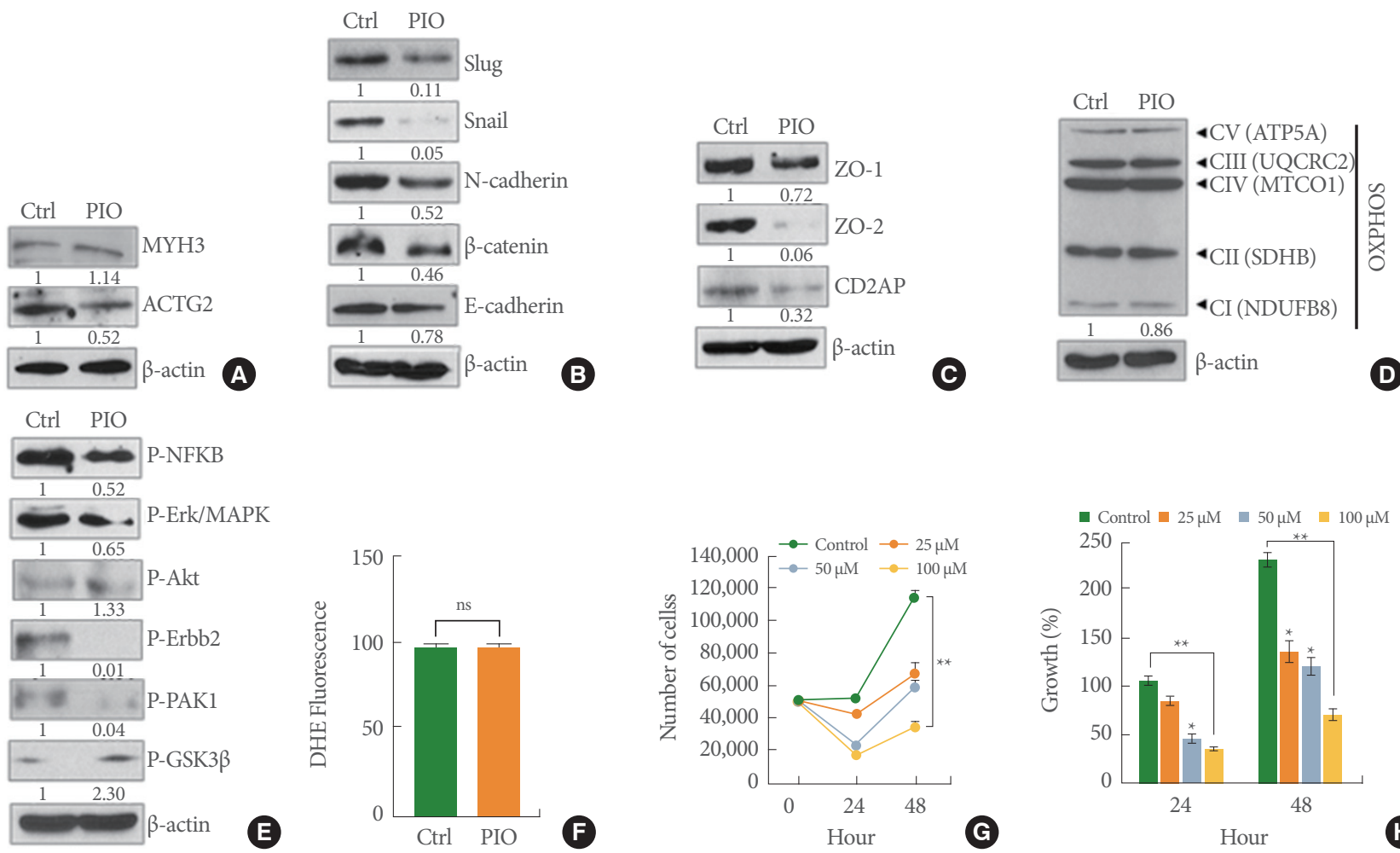

(

(D)

Fig. 5. Effects of pioglitazone treatment on THT-HU1 (human telomerase reverse transcriptase-immortalized human urothelial cells). Cells were incubated in $25 \mu \mathrm{M}$ of pioglitazone-containing Dulbecco's modified Eagle's medium for 72 hours. Whole-cell lysates were immunoblotted with various antibodies as indicated. (A) Immunoblot analyses were performed to validate the expression levels of identified differentially expressed proteins. The protein expression levels of MYH3 and ACTG2 significantly decreased with pioglitazone treatment (PIO), compared to controls (Ctrl). (B) Immunoblot analysis compared several epithelial-mesenchymal transition markers, including Slug, Snail, N-cadherin, $\beta$-catenin, and E-cadherin, in pioglitazone treated (PIO) and untreated (Ctrl) cells. (C) Cell junction markers (ZO-1, ZO-2, and CD2-associated protein) were also measured by immunoblot analysis. As the protein loading control, levels of $\beta$-actin were shown. (D) Levels of mitochondrial oxidative phosphorylation (OXPHOS) markers were compared between Ctrl and PIO groups. No significant changes were observed in response to pioglitazone treatment. (E) Western blot analysis of phospho-NF- $\mathrm{BB}$ (P-NFKB), phospho-Erk/MAPK (P-Erk/MAPK), phospho-HER2/ErbB2 (P-HER2/ErbB2), phospho-p21 activated kinase 1 (P-PAK1), and phospho-glycogen synthase kinase-3 $\beta$ (P-GSK3 $\beta$ ) were performed. $\beta$-actin was used as an internal control. (F) Reactive oxygen species production was compared between Ctrl and PIO groups. NS, nonsignificant. (G) Cell proliferation was quantified by trypan blue staining. ${ }^{* *} \mathrm{P}<0.005$. (H) Dose- and time-dependent cell growth rates were measured in an independent set of experiments. ${ }^{*} \mathrm{P}<0.01 .{ }^{*} \mathrm{P}<0.005$. NS, nonsignificant. ATP5A, ATP synthase subunit alpha; UQCRC2, ubiquinol-cytochrome C reductase core protein 2; MTCO1, mitochondrially encoded cytochrome C oxidase I; SDHB, succinate dehydrogenase; NDUFB8, NADH:ubiquinone oxidoreductase subunit B8. 
enchymal cell markers (N-cadherin, Snail, Slug, and $\beta$-catenin) were downregulated in response to pioglitazone treatment (Fig. $5 B$ ). In addition, tight junction proteins (zonula occludens [ZO] 1 and 2 and CD2-associated protein) that are involved in cellcell contact regions were greatly reduced in pioglitazone-treated cells (Fig. 5C).

To determine whether pioglitazone regulates mitochondrial quantity or metabolic respiration-associated proteins, mitochondrial OXPHOS proteins were measured via western blot analysis. The expression levels of OXPHOS subunits (C I subunit [NDUFB8], C II subunit [succinate dehydrogenase, SDHB], $\mathrm{C}$ III core protein 2 [ubiquinol- cytochrome $\mathrm{C}$ reductase core protein 2, UQCRC2], C IV subunit [mitochondrially encoded cytochrome C oxidase I, MTCO1], and C V alpha subunit [ATP synthase subunit alpha, ATP5A]) were not change by pioglitazone treatment (Fig. 5D). To profile the phosphorylation events in normal bladder cells, human TRT-HU1 cells were treated with or without pioglitazone. A series of western blot analyses showed that several phosphorylation events of specific signaling pathways were regulated by pioglitazone. The phosphorylation levels of NF-kB, Erk/MAPK, ERBB2, and PAK1 decreased. On the contrary, only the phosphorylation levels of GSK-3 $\beta$ increased. Additionally, previous studies have shown that the activation of PPAR $\gamma$ through agonists reduces diabetic myocardial fibrosis by transforming growth factor beta/extracellular signal-regulated kinase pathway and epithelial-mesenchymal transition [16], which were both found to be upregulated in pioglitazone-treated cells compared to controls (Fig. 5E).

To further elucidate on the role of pioglitazone on oxidative stress, ROS levels were measured and analyzed after 72-hour pioglitazone treatment. Levels of ROS were unchanged between the control and pioglitazone groups (Fig. 5F). In addition to our proteomic analysis, we assessed whether pioglitazone affects cell proliferation. The dose-dependent experiments in varying concentrations of pioglitazone $(25,50$, or $100 \mu \mathrm{M})$ for 24 and 48 hours, we noticed that the cell proliferation rates of cells treated for 48 hours were significantly suppressed, compared to controls (Fig. 5G, H).

\section{DISCUSSION}

There are many discrepancies surrounding the link between TZD usage and side effects on bladder function. Diabetic bladders can have several dysfunctions, ranging from bladder overactivity to impaired contractility, which represent a wide spec- trum of clinical symptoms. The widespread pathophysiology of bladder dysfunction in DM patients is associated with multiple factors like smooth muscle dysfunction, and urothelial impairment [17]. DM has been shown to be a significant predictor of death from cancer, including BC [18]. Evidence from a metaanalysis study has linked the use of pioglitazone to increased $\mathrm{BC}$ risk as well [13]. The tumorigenic effect of pioglitazone is thought to be due to signaling modifications in the PPAR pathway [19]. However, it is still unclear whether the long-term use of pioglitazone increases the chances of developing bladder dysfunctions or disease.

PPAR $\gamma$ is part of the PPAR subfamily that consists of 2 other subunits, alpha and beta/delta. As the most extensively studied subunit, the functions of PPAR $\gamma$ have been linked to several crucial biological processes, such as glucose metabolism, lipid biosynthesis, and anti-inflammatory response [20]. The association of PPAR $\gamma$ to diabetes is based on its role in lipid and glucose metabolism [21]. It has been shown that patients with dominant-negative mutations of PPAR $\gamma$ have severe hypoglycemia, insulin resistance, and DM [22]. This means that there is a significant genetic link between both PPAR $\gamma$ and DM. Treatment with TZDs has proven to substantially decrease insulin resistance in diabetes and possibly induces transcription of adipose cell differentiation, which generates smaller insulin-sensitive adipocytes [23]. However, despite their beneficial effects in patients with diabetes, TZDs are known to have adverse effects. This includes weight gain, fluid retention, bone mineral density reduction, and excess myocardial infarction [24]. The fluid retention induced by TZDs has also been proven to significantly increase urine volume and excretion of sodium [25]. Because of this, there is some controversy surrounding the safety of longterm TZD usage to treat diabetes.

In addition to the documented roles of PPAR $\gamma$ in lipid metabolism and $\mathrm{DM}$, there has been growing research on its functions in both $\mathrm{BC}$ and interstitial cystitis (IC). Approximately $20 \%-25 \%$ of human BC is driven by PPAR signaling, PPAR $\gamma$ gene amplification, or RXRA hot-spot mutations [26]. Furthermore, it was found that the PPAR $\gamma$ transcription pathway associated with an immune evasion in muscle-invasive BC [27]. Along with this immune escape, it has been proposed that PPAR $\gamma$ cooperates with the FOXA1 (forkhead box protein A1) and GATA3 (GATA binding protein 3) networks to drive transdifferentiation of basal BC cells into luminal types [28]. PPAR $\gamma$ has also been identified to be associated with IC. Mahal et al. [29] found that treating their IC rat model with PPAR $\gamma$ agonists 
improved bladder function and urinary health. Based on the complex relationship of PPAR $\gamma$ with diabetes and urological malignancies, it would be compelling to better understand how patients who routinely take TZDs are affected.

This study revealed that BMI1 is a master node in pioglitazone-regulated networks. Our proteomic profiling of BMI1 exhibited significant downregulation in normal bladder epithelial cells treated with pioglitazone, suggesting a potential inverse relationship between PPAR $\gamma$ and BMI1. One possible hypothesis for our future study is that PPAR $\gamma$ signaling induced by pioglitazone may cause loss of BMI1 expression in bladder cells. Expression of telomere maintenance proteins including HIST1H4A, HIST2H2AA3, and HIST2H2AC, which interact with $\mathrm{BMI}$, are downregulated as well. The presence of an upregulated telomere maintenance mechanism is a hallmark of cancer, and it is interesting to note that HIST2H2AA3 and HIST$2 \mathrm{H} 2 \mathrm{AC}$ were identified as genes upregulated, differentially expressed in BC [30]. This may signify a stage shift, by pioglitazone treatment, from developing cancer. PPAR $\gamma$ agonism has also been shown to increase the antitumor effects of histone deacetylase inhibitors in multiple myeloma cells [25].

Most notably, in our study, we found a series of contractile and cytoskeleton proteins, such as myosin light polypeptides, myosin heavy polypeptides, keratins, and ACTG2, as being significantly up- or downregulated in TRT-HU1 bladder epithelial cells treated with pioglitazone. Myosin is a particularly important structural component of muscles that interacts with actin filaments and plays a vital role in actin filament contraction, bundling, motor action, and binding. Furthermore, our network modeling revealed that these actin-binding proteins comprise the central hub. These results suggest the potential pathophysiological mechanisms of pioglitazone's effects.

Our data demonstrated that the activation of PPAR $\gamma$ signaling pathways suppressed the proliferation of bladder epithelial cells. Interestingly, these findings are consistent with clinical observations showing that the IC bladder has thinner epithelial layers [31] and that IC bladder cells exhibit reduced proliferation due to cell cycle arrest [32]. Our epigenetic remodeling study supports this mechanistic link between IC and defective cell proliferation [15], Furthermore, previous reports suggest that the antiproliferative role of PPAR $\gamma$ is through the upregulation of PTEN expression in various cancers, which leads to decreased PI-3K activity [33]. It is currently known that PPAR $\gamma$ agonists suppress cell cycling by downregulating cyclin D1 in several cancer cells; thereby, inhibiting cyclin D1/CDK-mediat- ed retinoblastoma $(\mathrm{pRb})$ phosphorylation and maintaining its active form to prevent the G1 to S phase transition [34]. Activation of PPAR $\gamma$ via agonists in normal human urothelial cells has been shown to link with late/terminal urothelial differentiation by protein induction of uroplakins, cytokeratins, and tight junction constituents [35].

We very much understand certain limitations of the study design arising from performing experiments in normal bladder cells, which cannot truly reflect the diabetic bladder condition [36,37]. Although additional experiments using bladder cells from diabetic condition are actually going on in our laboratory, it is out of scope of this study aiming to understand the pioglitazone effects on normal bladder cells. Another limitation of this study is that we used only one normal bladder cell line. This present study also suffers from limited impact on clinical application mainly due to in vitro experiments.

In summary, although pioglitazone is widely used among T2DM patients, its effects on the bladder are still in controversy. While there have been studies examining the effects of pioglitazone on healthy bladder cells, there is no conclusive evidence. Through unbiased quantitative proteomic profiling, our report comprehensively characterized the proteome of normal bladder epithelial cells treated with pioglitazone. We observed suppressed cell proliferation, which may be explained by the decreased expression of tight junction proteins. Taken together, our study provides no experimental evidence to support a tumorigenic effect of pioglitazone on the bladder. This proteomic approach will be useful for examining any potential side effects of the one of the most commonly used drugs.

\section{SUPPLEMENTARY MATERIALS}

Supplementary Tables 1 and 2 can be found via https://doi. org/10.5213/inj.1938186.093.

\section{AUTHOR CONTRIBUTION}

- Conceptualization: $J K$

- Formal Analysis: $M S, M K, A Y, P J$

- Investigation: $M S, M K$

- Methodology: MS, MK, AY, PJ

- Project Administration: JK, $W-K K, S Y$

- Writing - Original Draft: $J K, S Y$

- Writing - Review \& Editing: $J K$ 


\section{REFERENCES}

1. Guan Y, Zhang Y, Davis L, Breyer MD. Expression of peroxisome proliferator-activated receptors in urinary tract of rabbits and humans. Am J Physiol 1997;273:F1013-22.

2. Bodles AM, Varma V, Yao-Borengasser A, Phanavanh B, Peterson $\mathrm{CA}, \mathrm{McGehee} \mathrm{RE} \mathrm{Jr}$, et al. Pioglitazone induces apoptosis of macrophages in human adipose tissue. J Lipid Res 2006;47:2080-8.

3. Di Gregorio GB, Yao-Borengasser A, Rasouli N, Varma V, Lu T, Miles LM, et al. Expression of CD68 and macrophage chemoattractant protein-1 genes in human adipose and muscle tissues: association with cytokine expression, insulin resistance, and reduction by pioglitazone. Diabetes 2005;54:2305-13.

4. Yang SL, Wang JJ, Chen M, Xu L, Li N, Luo YL, et al. Pioglitazone use and risk of bladder cancer: an in vitro study. Int J Med Sci 2018; 15:228-37.

5. Lewis JD, Ferrara A, Peng T, Hedderson M, Bilker WB, Quesenberry CP Jr, et al. Risk of bladder cancer among diabetic patients treated with pioglitazone: interim report of a longitudinal cohort study. Diabetes Care 2011;34:916-22.

6. Belfiore A, Genua M, Malaguarnera R. PPAR- $\gamma$ agonists and their effects on IGF-I receptor signaling: Implications for cancer. PPAR Res 2009;2009:830501.

7. Necela BM, Su W, Thompson EA. Toll-like receptor 4 mediates cross-talk between peroxisome proliferator-activated receptor gamma and nuclear factor-kappaB in macrophages. Immunology 2008;125:344-58.

8. Fang H, Yao B, Yan Y, Xu H, Liu Y, Tang H, et al. Diabetes mellitus increases the risk of bladder cancer: an updated meta-analysis of observational studies. Diabetes Technol Ther 2013;15:914-22.

9. Lewis JD, Habel L, Quesenberry C, Mamtani R, Peng T, Bilker WB, et al. Proteinuria testing among patients with diabetes mellitus is associated with bladder cancer diagnosis: potential for unmeasured confounding in studies of pioglitazone and bladder cancer. Pharmacoepidemiol Drug Saf 2014;23:636-45.

10. Hampp C, Pippins J. Pioglitazone and bladder cancer: FDA's assessment. Pharmacoepidemiol Drug Saf 2017;26:117-8.

11. Chiu M, McBeth L, Sindhwani P, Hinds TD. Deciphering the roles of thiazolidinediones and PPAR $\gamma$ in bladder cancer. PPAR Res 2017;2017:4810672.

12. Li R, Metcalfe MJ, Ferguson JE 3rd, Mokkapati S, Nogueras González GM, Dinney CP, et al. Effects of thiazolidinedione in patients with active bladder cancer. BJU Int 2018;121:244-51.

13. Filipova E, Uzunova K, Kalinov K, Vekov T. Pioglitazone and the risk of bladder cancer: a meta-analysis. Diabetes Ther 2017;8:705-
26.

14. Garry EM, Buse JB, Lund JL, Pate V, Stürmer T. Comparative safety of pioglitazone versus clinically meaningful treatment alternatives concerning the risk of bladder cancer in older US adults with type 2 diabetes. Diabetes Obes Metab 2018;20:129-40.

15. Shahid M, Gull N, Yeon A, Cho E, Bae J, Yoon HS, et al. Alpha-oxoglutarate inhibits the proliferation of immortalized normal bladder epithelial cells via an epigenetic switch involving ARID1A. Sci Rep 2018;8:4505.

16. Yan XL, Wang YY, Yu ZF, Tian MM, Li H. Peroxisome proliferatoractivated receptor-gamma activation attenuates diabetic cardiomyopathy via regulation of the TGF- $\beta$ /ERK pathway and epithelial-tomesenchymal transition. Life Sci 2018;213:269-78.

17. Gomez CS, Kanagarajah P, Gousse AE. Bladder dysfunction in patients with diabetes. Curr Urol Rep 2011;12:419-26.

18. Coughlin SS, Calle EE, Teras LR, Petrelli J, Thun MJ. Diabetes mellitus as a predictor of cancer mortality in a large cohort of US adults. Am J Epidemiol 2004;159:1160-7.

19. Peters JM, Shah YM, Gonzalez FJ. The role of peroxisome proliferator-activated receptors in carcinogenesis and chemoprevention. Nat Rev Cancer 2012;12:181-95.

20. Mansure JJ, Nassim R, Kassouf W. Peroxisome proliferator-activated receptor gamma in bladder cancer: a promising therapeutic target. Cancer Biol Ther 2009;8:6-15.

21. Kim HI, Ahn YH. Role of peroxisome proliferator-activated receptor-gamma in the glucose-sensing apparatus of liver and beta-cells. Diabetes 2004;53 Suppl 1:S60-5.

22. Barroso I, Gurnell M, Crowley VE, Agostini M, Schwabe JW, Soos $\mathrm{MA}$, et al. Dominant negative mutations in human PPARgamma associated with severe insulin resistance, diabetes mellitus and hypertension. Nature 1999;402:880-3.

23. Spiegelman BM. PPAR-gamma: adipogenic regulator and thiazolidinedione receptor. Diabetes 1998;47:507-14.

24. Wang S, Dougherty EJ, Danner RL. PPAR $\gamma$ signaling and emerging opportunities for improved therapeutics. Pharmacol Res 2016;111: $76-85$

25. Zhou L, Liu G, Jia Z, Yang KT, Sun Y, Kakizoe Y, et al. Increased susceptibility of $\mathrm{db} / \mathrm{db}$ mice to rosiglitazone-induced plasma volume expansion: role of dysregulation of renal water transporters. Am J Physiol Renal Physiol 2013;305:F1491-7.

26. Halstead AM, Kapadia CD, Bolzenius J, Chu CE, Schriefer A, Wartman LD, et al. Bladder-cancer-associated mutations in RXRA activate peroxisome proliferator-activated receptors to drive urothelial proliferation. Elife 2017;6:e30862.

27. Korpal M, Puyang X, Jeremy Wu Z, Seiler R, Furman C, Oo HZ, et 
al. Evasion of immunosurveillance by genomic alterations of PPAR $\gamma /$ RXRa in bladder cancer. Nat Commun 2017;8:103.

28. Warrick JI, Walter V, Yamashita H, Chung E, Shuman L, Amponsa VO, et al. FOXA1, GATA3 and PPAR $\gamma$ cooperate to drive luminal subtype in bladder cancer: a molecular analysis of established human cell lines. Sci Rep 2016;6:38531.

29. Mahal A, Young-Lin N, Dobberfuhl A, Estes J, Comiter CV. Peroxisome proliferator-activated receptor gamma agonist as a novel treatment for interstitial cystitis: a rat model. Investig Clin Urol 2018;59:257-62.

30. Han Y, Jin X, Zhou H, Liu B. Identification of key genes associated with bladder cancer using gene expression profiles. Oncol Lett 2018;15:297-303.

31. Keay S, Zhang CO, Shoenfelt JL, Chai TC. Decreased in vitro proliferation of bladder epithelial cells from patients with interstitial cystitis. Urology 2003;61:1278-84.

32. Kim J, Keay SK, Dimitrakov JD, Freeman MR. p53 mediates interstitial cystitis antiproliferative factor (APF)-induced growth inhibition of human urothelial cells. FEBS Lett 2007;581:3795-9.

33. Patel L, Pass I, Coxon P, Downes CP, Smith SA, Macphee CH. Tu- mor suppressor and anti-inflammatory actions of PPARgamma agonists are mediated via upregulation of PTEN. Curr Biol 2001;11: 764-8.

34. Qin C, Burghardt R, Smith R, Wormke M, Stewart J, Safe S. Peroxisome proliferator-activated receptor gamma agonists induce proteasome-dependent degradation of cyclin D1 and estrogen receptor alpha in MCF-7 breast cancer cells. Cancer Res 2003;63:958-64.

35. Varley CL, Stahlschmidt J, Smith B, Stower M, Southgate J. Activation of peroxisome proliferator-activated receptor-gamma reverses squamous metaplasia and induces transitional differentiation in normal human urothelial cells. Am J Pathol 2004;164:1789-98.

36. Tomechko SE, Liu G, Tao M, Schlatzer D, Powell CT, Gupta S, et al. Tissue specific dysregulated protein subnetworks in type 2 diabetic bladder urothelium and detrusor muscle. Mol Cell Proteomics 2015;14:635-45.

37. Yohannes E, Chang J, Christ GJ, Davies KP, Chance MR. Proteomics analysis identifies molecular targets related to diabetes mellitus-associated bladder dysfunction. Mol Cell Proteomics 2008;7:1270-85. 